\title{
57. La reformulation comme stratégie d'apprentissage du FLE: exemple du manuel Panorama 4
}

\section{Özge SÖNMEZ1}

APA: Sönmez, Ö. (2021). La reformulation comme stratégie d'apprentissage du FLE: exemple du manuel Panorama 4. RumeliDE Dil ve Edebiyat Araştırmaları Dergisi, (24), 1004-1028. DOI: 10.2900o/rumelide.995469.

\section{Résumé}

L'acte de reformulation continue à être l'objet de recherche des linguistes aussi bien que les didacticiens. Les méthodes d'enseignement des langues étrangères qui se développent en fonction de l'évolution des besoins, des progrès technologiques et des progrès scientifiques prennent en compte l'acte de reformulation comme un moyen d'apprentissage des compétences de langue étrangères. Dans l'apprentissage des langues, l'étudiant doit acquérir de nombreuses compétences. Bien que ces compétences soient définies de différentes manières selon les méthodes pédagogiques 4 compétences principales sont généralement mentionnées: la compréhension écrite, l'expression écrite, la compréhension orale et l'expression orale. Un enseignant de langues étrangères applique de nombreuses techniques pour aider les étudiants à acquérir ces compétences dans une langue. L'une d'elles est la reformulation. L'enseignant fait différentes reformulations, consciemment ou non, en donnant ses cours, en parlant, en posant des questions aux étudiants, en répondant, voire en organisant certaines informations au tableau. Les reformulations ont également une place entière dans les unités des manuels et dans les activités proposées aux étudiants. Dans notre étude, nous analyserons dans quelle mesure la reformulation, qui est en fait une créativité linguistique, sert dans le manuel «Panorama 4 " niveau B2, quelles compétences linguistiques ont été proposées aux étudiants par le biais des activités linguistiques, quels types de reformulations elles contiennent et les fonctions de ces reformulations.

Mots-clés: Acte de reformulation, compétences des langues, approche communicative, Panorama 4

\section{Reformulation as a learning strategy for French as a foreign language: example from the "Panorama 4" manual}

\begin{abstract}
The act of reformulation continues to be the subject of research among linguists as well as educationalists. Foreign language teaching methods which develop in line with changing needs, technological progress and scientific progress take into account the act of reformulation as a means of learning foreign language skills. In language learning, the student must acquire many skills. Although these skills are defined in different ways according to the teaching methods, 4 main skills are generally mentioned: reading comprehension, written expression, oral comprehension and oral expression. A foreign language teacher applies many techniques to help students acquire these skills in a language. One of them is rephrasing. The teacher makes various reformulations, consciously or not, by giving his lessons, by speaking, by asking questions to the students, by answering, even by organizing certain information on the board. Reformulations also have a full place in the units of
\end{abstract}

Dr. Öğr. Üyesi, Dokuz Eylül Üniversitesi, Buca Eğitim Fakültesi, Fransız Dili Eğitimi ABD (İzmir, Türkiye), osonmez@yahoo.fr, ORCID ID: 0ooo-0oo1-6515-6864 [Araştırma makalesi, Makale kayıt tarihi: 26.07.2021-kabul tarihi: 20.09.2021; DOI: 10.29000/rumelide.995469]

RumeliDE Dil ve Edebiyat Araştırmaları Dergisi Osmanağa Mahallesi, Mürver Çiçeği Sokak, No:14/8 Kadıköy - ISTANBUL / TÜRKIYE 34714 e-posta: editor@rumelide.com tel: +90 $5057958124,+902167730616$

Address

RumeliDE Journal of Language and Literature Studies

Osmanağa Mahallesi, Mürver Çiçeği Sokak, No:14/8

Kadıköy - ISTANBUL / TURKEY 34714

e-mail: editor@rumelide.com,

phone: +90 5057958124 , +90 2167730616 
textbooks and in the activities offered to students. In our study, we will analyze to what extent the reformulation, which is in fact a linguistic creativity, is used in the manual "Panorama 4" level B2, what linguistic skills were offered to the students through the linguistic activities, what types of reformulations they contain and the functions of these reformulations.

Keywords: Reformulation act, language skills, communicative approach, Panorama 4

\title{
Fransızca öğretiminde bir öğrenme stratejisi olarak “yeniden söyleyim”: Panorama 4 örneği
}

\begin{abstract}
Öz
"Yeniden söyleyim" (fr. la reformulation) eylemi, eğitimciler kadar dilbilimciler arasında da araştırma konusu olmaya devam etmektedir. Değişen ihtiyaçlar, teknolojik ilerlemeler ve bilimsel ilerlemeler doğrultusunda gelişen yabancı dil öğretim yöntemleri, yabancı dil becerilerini öğrenmenin bir aracı olarak "yeniden söyleyim” (fr. la reformulation) eylemini dikkate almaktadır. Dil öğreniminde öğrencinin birçok beceri kazanması gerekir. Bu beceriler öğretim yöntemlerine göre farklı şekillerde tanımlansa da genel olarak 4 ana beceriden bahsedilmektedir: okuduğunu anlama, yazılı anlatım, sözlü anlama ve sözlü anlatım. Bir yabancı dil öğretmeni, öğrencilere bu becerileri bir dilde kazandırmak için birçok teknik uygular. Bunlardan biri yeniden ifade etmektir. Öğretmen, derslerini vererek, konuşarak, öğrencilere sorular sorarak, cevaplayarak, hatta bazı bilgileri tahtada düzenleyerek, bilinçli veya bilinçsiz olarak çeşitli yeniden söyleyimler yapar. Ders kitaplarının ünitelerinde ve öğrencilere sunulan etkinliklerde de yeniden söyleyimlerin yeri vardır. Ülkemizde Fransızca öğretimi alanında dilin çeşitli seviyelerinde eylem odaklı yaklaşıma dayalı birçok ders kitabı kullanılmaktadır ancak bu ders kitapları kullanıldı̆̆ında öğrencilerden beklenen görevlerin çoğu benzetime (fr. la simulation) dönüşmektedir. Benzetim iletişimsel yaklaşımın temel tekniğidir. $\mathrm{Bu}$ neredeyse kaçınılmazdır çünkü öğrencilerimiz günlük yaşamlarında çok nadiren Fransızca konuşma firsatı bulurlar. Çalışmamızda ders kitabı olarak Panorama 4’ü seçmemizin temel nedeni iletişimsel yaklaşıma yönelik olmasıdır. Çalışmamızda aslında bir dilsel yaratıcılık olan "yeniden söyleyim”in "Panorama 4" B2 düzeyindeki ders kitabında ne ölçüde kullanıldığını, dil etkinlikleriyle öğrencilere hangi dil becerilerinin kazandırıldığını, hangi tür “yeniden söyleyim”ler yapıldığını ve bu söyleyimlerin işlevlerini inceleyeceğiz.
\end{abstract}

Anahtar kelimeler: Yeniden söyleyim, dil becerileri, iletişimsel yaklaşım, Panorama 4

\section{Introduction}

Les méthodes d'enseignement des langues étrangères se développent en fonction de l'évolution des besoins, des progrès technologiques et des progrès scientifiques. Si l'on prend en compte les méthodologies utilisées dans l'enseignement des langues étrangères dans notre pays, il est possible de faire ressortir deux approches particulièrement prépondérantes. L'une d'elles est l'approche communicative, l'autre, l'approche actionnelle. Dans les pays non francophones comme la Turquie, l'approche communicative est a priori la plus souvent utilisée. Pour cette raison, les manuels élaborés selon l'approche communicative sont préférés par les enseignants de langues étrangères. Les raisons en sont variées. En tant qu'exigence des manuels basés sur l'approche actionnelle et des principes de cette approche ; apprendre une langue étrangère par la pratique, basée sur des expériences dans la vie réelle, il s'agit de conforter les étudiants à effectuer principalement des tâches micro-macro hors classe, des

\footnotetext{
\begin{tabular}{r|l} 
Adres & Address \\
RumeliDE Dil ve Edebiyat Araştırmaları Dergisi & RumeliDE Journal of Language and Literature Studies
\end{tabular} Osmanağa Mahallesi, Mürver Çiçeği Sokkak, No:14/8 $\quad$ Osmanağa Mahallesi, Mürver Çiçeği Sokak, No:14/8 Kadıköy - ÍSTANBUL / TÜRKIYE 34714 Kadıköy - ISTANBUL / TURKEY 34714 e-posta: editor@rumelide.com e-mail: editor@rumelide.com, tel: +90 505 7958124, +90 2167730616 phone: +90 505 7958124, +90 2167730616
} 
projets et des scénarios qui nécessitent une utilisation efficace du français. Dans le domaine de l'enseignement du français de notre pays, de nombreux manuels basés sur l'approche actionnelle sont utilisés à plusieurs niveaux de la langue, mais lorsque ces manuels sont utilisés, la plupart des tâches attendues de la part des étudiants se transforment en simulation, qui est pourtant la technique de base de l'approche communicative. C'est presque inévitable car nos étudiants n'ont que très rarement l'occasion de parler en français dans leur vie quotidienne. C'est la raison par laquelle nous avons choisi le manuel d'apprentissage Panorama 4 comme objet d'étude de ce travail vu qu'il est particulièrement tourné vers l'approche communicative. Panorama 4 est un manuel élaboré pour le niveau B2 selon le CECRL (Cadre Européen de Référence pour les Langues), pour les adolescents et les adultes.

Dans l'apprentissage des langues, l'étudiant est invité à acquérir de nombreuses compétences. Bien que ces compétences soient définies de différentes manières selon les méthodes pédagogiques 4 compétences principales sont généralement mentionnées : la compréhension écrite, l'expression écrite, la compréhension orale et l'expression orale. Un enseignant de langues étrangères applique de nombreuses techniques pour aider les étudiants à acquérir ces compétences dans une langue. L'une d'elles est la reformulation. L'enseignant fait différentes reformulations, consciemment ou non, en donnant ses cours, en parlant, en posant des questions aux étudiants, en répondant, voire en organisant certaines informations au tableau. Les reformulations ont une place entière dans les unités des manuels et dans les activités proposées aux étudiants. Dans notre étude, nous analyserons dans quelle mesure la reformulation, qui est en fait une créativité linguistique, sert dans le manuel " Panorama 4 ", quelles compétences linguistiques ont été proposées aux étudiants par le biais des activités linguistiques, quels types de reformulations elles contiennent et les fonctions de ces reformulations.

\section{« La reformulation » au carrefour de plusieurs disciplines}

Le Larousse nous dit : «Formuler de nouveau et d'une manière plus correcte » Si l'on s'en tient à cette définition alors nous comprenons que le locuteur qui a déjà formulé une phrase est invité à la reformuler de manière plus correcte ou plus compréhensible. En nous basant sur l'idée que les signes linguistiques sont des codes qui aident à concrétiser le monde réel dans le monde linguistique par l'abstraction, on peut soutenir que l'existence de l'acte de reformulation chez l'individu est apparue depuis que l'homme a acquis la capacité d'exprimer ses pensées par le biais de la langue. Cet acte de reformulation qui n'est pas du tout nouveau, a été repris et étudié davantage par des linguistes. La reformulation est définie comme «Tout processus de reprise d'un énoncé antérieur qui maintient, dans l'énoncé reformulé, une partie invariante à laquelle s'articule le reste de l'énoncé, partie variante à l'énoncé source » (Martinot, 1994 : 65). Dans le schéma de « fonctions du langage » proposé par Roman Jacobson dans son livre «Essai de linguistique générale » daté de 1963, la fonction métalinguistique, c'est-à-dire lorsque la langue est centrée sur le code, la reformulation est en scène. Par exemple, des livres de grammaire, des dictionnaires mettent en œuvre la fonction métalinguistique en donnant des définitions, en faisant des classifications des éléments linguistiques. Cela ne signifie pas que les autres fonctions du langage, de Jakobson, les fonctions : émotive, conative, référentielle, phatique et poétique n’impliquent pas d'acte de reformulation. La langue en tant que «système» a, elle-même, une nature basée sur l'acte de reformulation. Elle est fréquemment produite par un sujet parlant dans la langue orale, a fait l'objet de recherches sur la langue maternelle, sur la langue étrangère et sur le domaine de traduction. La classification Jacobsonienne divise la traduction en trois catégories :

« -La traduction intralinguale ou reformulation, qui consiste en l'interprétation des signes linguistiques au moyen d'autres signes de la même langue.

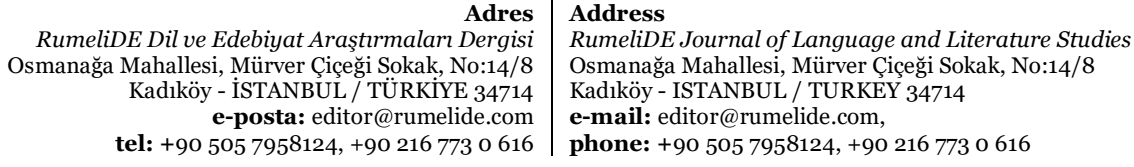


-La traduction interlinguale ou traduction proprement dite, qui consiste en l'interprétation des signes linguistiques au moyen d'autres signes d'une autre langue.

-La traduction intersémiotique ou transmutation, qui consiste en l'interprétation des signes linguistiques au moyen de systèmes de signes non linguistiques. » (Jacobson, 2003 :79)

Jacobson adopte une approche sémiotique du phénomène de la traduction, affirmant qu'il ne serait jamais possible de parvenir à une équivalence complète entre des mots de différentes langues, donc cette diffusion provient d'une expression indirecte. La traduction intralinguale n'est en fait qu'une étape de la traduction proprement dite, c'est l'étape où on analyse des signes linguistiques de texte source et où on donne un texte reproduit conforme au texte source.

La recherche sur la langue maternelle examine comment, en particulier, les jeunes enfants bénéficient de cet acte tout en acquérant leur langue maternelle. On étudie comment les enfants acquièrent leur langue maternelle selon leurs catégories d'âge et comment ils changent l'énoncé initial. Il existe de nombreuses études sur ce sujet dans le domaine de la traduction. Des études sur l'enseignement des langues étrangères ont souvent été menées sur la langue orale utilisée par l'enseignant ou l'élève en classe. Les recherches sont souvent basées sur l'analyse des enregistrements de cours réalisés dans la classe et la transcription de ces enregistrements par des perspectives linguistiques, syntaxiques, sémantiques, lexicales, etc.

Dans les études actuelles, les gestes, les mimiques, les schémas que l'enseignant dessine au tableau et les différents supports visuels qu'il utilise, ainsi que le langage verbal et écrit utilisé par l'enseignant dans la classe, sont considérés comme un acte de reformulation. Les transitions entre ces langages créés selon des codes différents constituent également des données importantes pour les chercheurs qui étudient l'acte de reformulation. De même que celle-ci « fonctionne comme un puissant outil pédagogique (...) une nouvelle formulation pour faire passer des contenus, en les rendant plus accessibles (recours à un lexique de base plus fréquent, avec une formulation plus analytique), plus concrets, plus proches de connaissances disponibles». (Noyau, 2010: 556) Dans le domaine de la didactique des langues étrangères, les manuels utilisés sont considérés comme aussi importants que l'enseignant lui-même, pour l'apprentissage de la langue cible. Par conséquent, bien que le manuel soit un objet, il doit être considéré comme un support linguistique à part entière, en particulier pour les étudiants des pays non francophones comme notre pays.

«Les démarches didactiques axées sur la reformulation en tant que stratégie d'apprentissage sont censées remplir des fonctions compensatoires et en même temps stimuler le développement des compétences en langue. Elles permettent de diagnostiquer les difficultés d'acquisition du langage et de rendre plus transparent (et par conséquent plus facile) l'apprentissage d'une langue étrangère. " (Karpiska-Szaj et Paprocka-Pıtrowska, $2014: 58$ )

\section{«La reformulation » comme stratégie d'apprentissage dans le manuel de Panorama 4}

Le manuel Panorama 4 que nous avons utilisé dans notre étude, a été rédigé conformément pour le niveau B2. Ce livre comprend 6 unités, basées sur 4 compétences langagières. Chaque unité est classée en tant que thème et des tâches sont assignées aux étudiants. Dans chaque unité, on trouve une grande variété de documents authentiques, les apprenants ont à leur disposition une boîte à outils et une partie du vocabulaire est également fournie avant l'activité d'écoute. Afin de préciser les types de reformulation dans le manuel et la manière dont elles sont abordées, nous avons divisé notre étude en 3 parties.

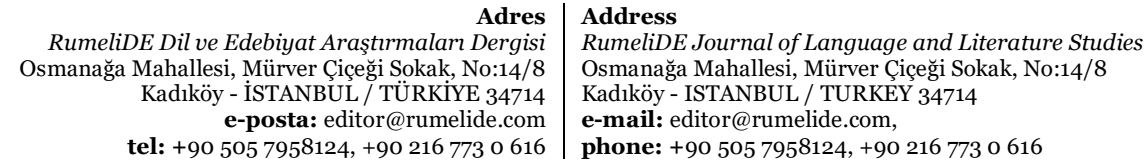

RumeliDE Dil ve Edebiyat Araştırmaları Dergis tel: +90 505 7958124, +902167730616 
Premièrement, nous avons classé les activités que l'étudiant est censé réaliser lors de l'acte de reformulation explicitement ou implicitement dans chaque unité. Dans cette classification, nous avons identifié 9 verbes dans les consignes des exercices. Ensuite, nous avons classé toutes ces activités selon 4 compétences langagières. Notre objectif dans cette classification est de déterminer quelle compétence de langue a été choisie dans l'activité comme point de départ de l'acte de reformulation et quelle compétence de langue a été activée à la fin de cette activité. Nous avons également examiné les démarches suivies lors de ces transitions entre les compétences de langue. Nous avons identifié 5 démarches principales lors de l'acte de reformulation.

Dans l'étape suivante, nous avons classé les reformulations utilisées dans les boîtes à outils disponibles dans le manuel, qui est un des outils fournis par celui-ci. Dans cette classification que nous avons réalisée pour chaque unité, nous avons identifié 5 démarches de reformulation. Dans la dernière étape, nous avons traité la partie de l'enseignement du vocabulaire dans chaque unité du manuel. Dans cette partie, nous avons, également, identifié 5 méthodes de reformulation.

Avant de passer à une analyse détaillée, abordons dans un premier temps sur les définitions des 9 verbes qui incluent implicitement ou explicitement un acte de reformulation tel que prévu dans les consignes des activités. Ces verbes sont : « résumer», «réécrire», «rédiger», «formuler », « reformuler», « regrouper », « définir », « faire une synthèse » et « faire une analyse ».

Le verbe « résumer », a trois définitions à peu près similaires. La première est : « Exprimer sous une forme plus brève ce qui a été dit ou écrit ». Cette définition consiste à raccourcir le texte source (oral ou écrit) et à produire un autre texte (oral ou écrit) sans utiliser de méthodes linguistiques strictes. Quand on choisit de faire ce type de résumé, on a recours à la simplification ou à la généralisation du texte source. Quant à la deuxième définition, « présenter un ensemble en le réduisant à l'essentiel », elle est également associée à la simplification, mais cette fois-ci, on suit une méthode stricte dans laquelle les informations jugées inutiles ou superflues, sont éliminées. La dernière définition est " présenter, renfermer en soi les caractères principaux de quelque chose ». Elle met l'accent sur la sélection des idées de base du texte source et reformule en tenant compte de ces idées.

Le verbe « réécrire » a deux définitions. La première définition est "rédiger quelque chose sous une nouvelle forme ", et cela concerne une reformulation qui vise au renouvellement d'un texte source. La deuxième définition « Reprendre l'écriture d'un texte en améliorant la forme ou en l'adaptant à une nouvelle destination. ", est un acte de reformulation qui cherche un perfectionnement, une progression dans le texte reproduit. En général, la réécriture se fait fréquemment par l'adaptation, par complexification ou par transposition du texte source. Il est à remarquer ici que cet acte de reformulation nécessite plus de créativité que le résumé qui se base sur la simplification d'un texte ou la sélection des idées principales. " Re- » dénote la répétition d'un événement qui efface le premier et, dans le même temps, l'existence de ses deux états successifs. » (Henry $2016: 27$ )

Le verbe « rédiger » est défini dans le dictionnaire comme " écrire un texte dans une forme élaborée et en respectant les règles du genre auquel il appartient ». Dans cette définition, il est clair que cet acte de reformulation implique une méthodologie stricte et bien définie, car elle nous oblige à suivre certaines règles linguistiques textuelles. "Rédiger » (...) c'est sans doute à la fois exprimer une deuxième fois ce qui est contenu dans une documentation de départ et l'écrire autrement et mieux. » (Henry $2016: 28$ )

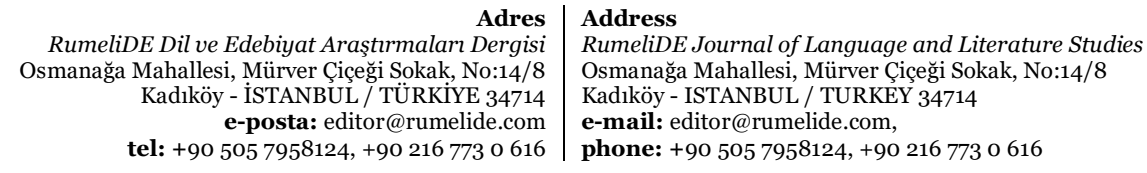


Le verbe "formuler" est défini comme « rédiger un texte, un énoncé selon la formule adéquate, d'une certaine manière ». L'acte de formulation peut être considéré comme une création proprement dite, c'està-dire, qu'il ne provient pas nécessairement d'un texte source (d'un texte étendu), mais par contre pour formuler un tel texte quel que soit son sujet, il faut avoir au moins des prérequis linguistiques et un bagage culturel suffisant pour se permettre de considérer un texte virtuel et omniprésent. Cette définition nous montre que cet acte de reformulation nécessite une rédaction personnelle tout en étant fidèle à des règles linguistiques. Cet acte peut être réalisé par l'adaptation, l'imitation des prérequis, par la simplification ou par le replacement imitatif des structures linguistiques.

Le verbe « reformuler » est défini comme « formuler de nouveau et d'une manière plus correcte. » Cette définition repose sur l'amélioration d'un texte source selon certaines règles précises et strictes. Il ne s'agit pas d'une reformulation libre et vaste. Ceci peut être réalisé par la simplification, la complexification, l'adaptation, le replacement, etc.

« «Réécrire » ou « reformuler » indiquent qu'un texte ou un énoncé existant a fait l'objet d'une reprise, mais également que la deuxième version s'ajoute à la première. Il ne s'agit pas seulement de produire une nouvelle copie qui annule la première. Une autre valeur couramment attribuée à « re- » est celle d'intensification, de dépassement. Dans cette perspective, « reformuler » ou " réécrire » ne dénotent pas seulement la production d'une deuxième version purement synonymique d'un énoncé ou d'un texte, mais aussi celle d'une version qui dépasse la première en la corrigeant ou en l'améliorant. » (Henry, $2016: 27)$

Le verbe "regrouper" est défini comme " mettre ensemble des éléments qui étaient distincts pour former un tout ». Cette définition consiste à faire une réunification, un replacement afin de créer un ensemble. Cet acte peut être réalisé par un replacement imitatif ou créatif.

Une définition du verbe « définir » est « énoncer la nature, les qualités, les propriétés essentielles de l'être ou de la chose que le mot désigne ; donner sa définition ». Une définition peut être considérée comme une explication détaillée, une paraphrase de la notion en question. C'est donner un sens quasi-équivalent car il est impossible de parler d'une synonymie absolue.

Faire une synthèse, c'est faire une " opération intellectuelle par laquelle on réunit en un tout cohérent, structuré et homogène divers éléments de connaissance concernant un domaine particulier." Cet acte consiste à faire un ensemble logique et cohérent d'éléments linguistiques. Cette forme de combinaison est aussi une reformulation nécessitant du raisonnement et un travail précis. « Les tâches à accomplir ici sont beaucoup plus complexes et mettent chacune en œuvre différents savoir-faire dont on exige la maîtrise et qui vont au-delà d'une bonne connaissance de la langue. » (Cuq, 2005 : 450)

De même, faire une analyse est "une action de résumer un texte en le décomposant en ses éléments essentiels ; résultat de cette action. » Cette action se base sur la différenciation des éléments principaux d'un texte. Ceci peut être accepté comme un résumé, mais pas par la simplification comme le résumé proprement dit, c'est fait par la complexification. « Ce sont des exercices de contraction de texte(s) et de reformulation objective qui engagent non seulement la compréhension des documents de l'expression écrite, mais également la connaissance des règles méthodologique. » (Cuq et Gruca, 2005 : 450).

\section{Panorama 4-Unité 1}

La première unité qui est nommée «Idées » est divisée en 4 parties. Ces 4 parties qui sont d'ailleurs communes dans toutes les autres unités du manuel sont: "Analyses et Commentaires ", "DossierDébat », "Simulation » et " Projet ». Cette première unité du manuel se concentre sur des objectifs

Adres | Address

RumeliDE Dil ve Edebiyat Araştırmalar Dergisi $\quad$ RumeliDE Journal of Language and Literature Studies Osmanağa Mahallesi, Mürver Çiçeği Sokak, No:14/8 $\quad$ Osmanağa Mahallesi, Mürver Çiçeği Sokak, No:14/8 Kadıköy - ÍSTANBUL / TÜRKIYE 34714 Kadıköy - ISTANBUL / TURKEY 34714 e-posta: editor@rumelide.com e-mail: editor@rumelide.com, tel: +90 505 7958124, +90 2167730616 phone: +90 505 7958124, +90 2167730616 
comme : «Formuler les idées ou les informations principales d'un document. », « Regrouper des informations. », " Convaincre, accuser, se défendre, éluder une question. », « Définir une notion. », « Expression des droits et des devoirs. », « Faire des propositions et des suggestions », etc. Cette unité représente un total de 26 activités. 8 de ces 26 activités sont consacrées à l'acte de reformulation par l'utilisation des différentes compétences (la compréhension orale (CO), la compréhension écrite (CE), la production orale (PO) et la production écrite (PE) de langue. Afin de mieux clarifier notre classification sur cette unité, nous avons le tableau suivant :

Tableau 1 : Unité 1

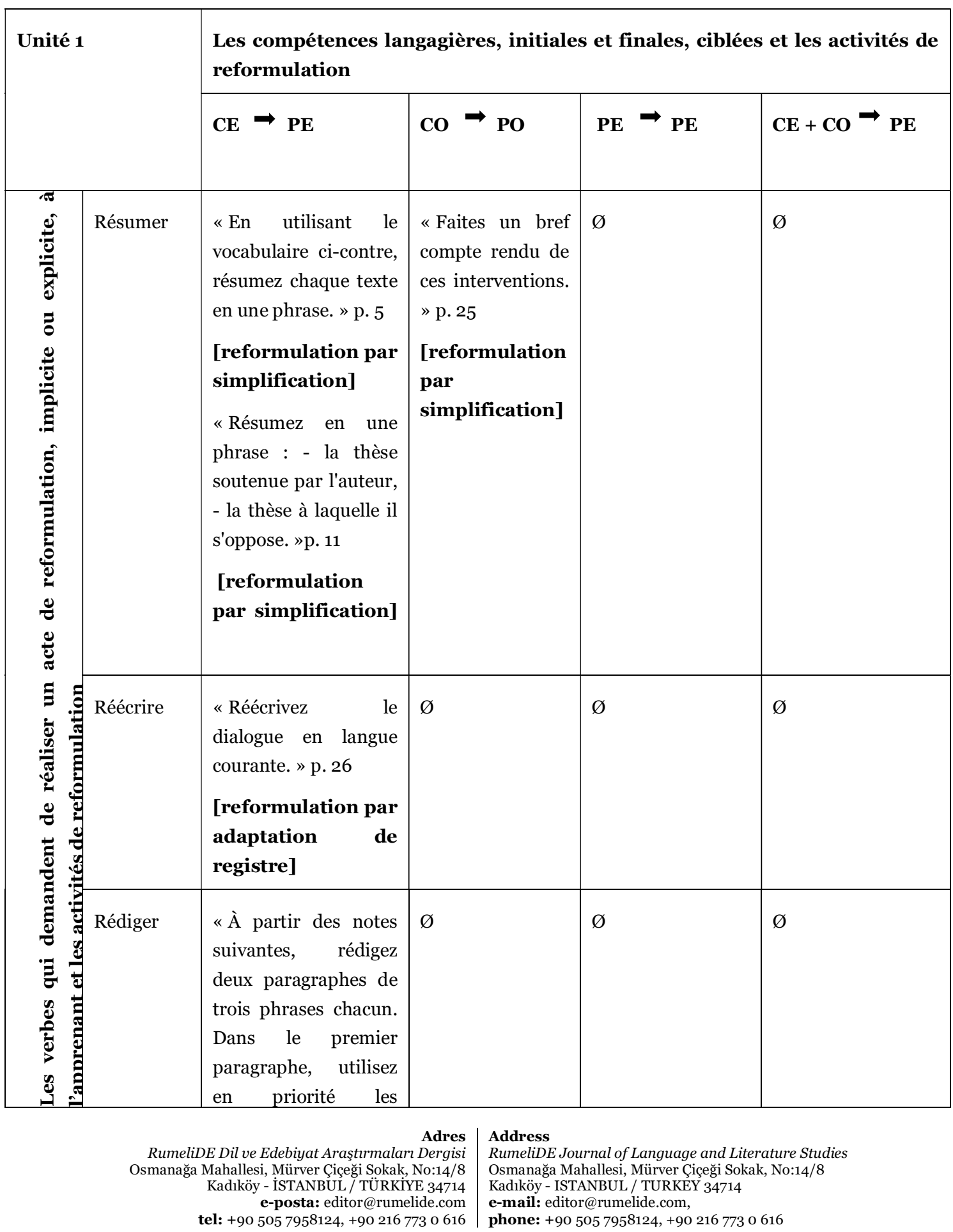




\begin{tabular}{|c|c|c|c|c|}
\hline & 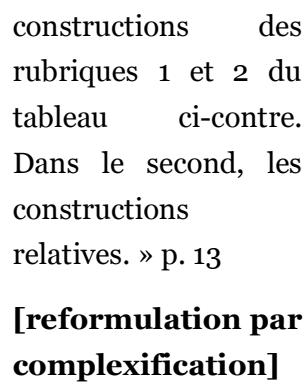 & & & \\
\hline Formuler & $\begin{array}{l}\text { «Formulez I'idée } \\
\text { principale développée } \\
\text { par Michel Lacroix. » } \\
\text { p. } 7 \\
\text { [reformulation par } \\
\text { simplification] }\end{array}$ & $\varnothing$ & $\varnothing$ & $\varnothing$ \\
\hline Reformuler & $\varnothing$ & $\varnothing$ & $\varnothing$ & $\varnothing$ \\
\hline Regrouper & $\varnothing$ & $\varnothing$ & $\begin{array}{l}\text { "Regroupez dans } \\
\text { le tableau suivant } \\
\text { l'ensemble des } \\
\text { idées et des } \\
\text { exemples que vous } \\
\text { avez trouvés } \\
\text { depuis l'exercice } \\
\text { 1. »p. } 15 \\
\text { [Reformulation } \\
\text { par un } \\
\text { replacement } \\
\text { imitatif] }\end{array}$ & $\begin{array}{l}\text { «En lisant le } \\
\text { dossier ci-dessus et } \\
\text { en écoutant le } \\
\text { document sonore } \\
\text { préparez une fiche } \\
\text { sur la condition des } \\
\text { femmes en France. } \\
\text { Regroupez les } \\
\text { principales } \\
\text { informations } \\
\text { autour des points } \\
\text { suivants (utilisez si } \\
\text { possible des formes } \\
\text { nominales). »p. 17 } \\
\text { [Reformulation } \\
\text { par } \\
\text { replacement un } \\
\text { créatif] }\end{array}$ \\
\hline Définir & $\varnothing$ & $\varnothing$ & $\varnothing$ & $\varnothing$ \\
\hline $\begin{array}{l}\text { Faire une } \\
\text { synthèse }\end{array}$ & $\varnothing$ & $\varnothing$ & $\varnothing$ & $\varnothing$ \\
\hline
\end{tabular}

RumeliDE Dil ve Edebiyat Araştırmaları Dergisi Osmanağa Mahallesi, Mürver Çiçeği Sokak, No:14/8 Kadıköy - ISTANBUL / TÜRKIYE 34714 e-posta: editor@rumelide.com tel: +90 $5057958124,+902167730616$
Address

RumeliDE Journal of Language and Literature Studies Osmanağa Mahallesi, Mürver Çiçeği Sokak, No:14/8

Kadıköy - ISTANBUL / TURKEY 34714

e-mail: editor@rumelide.com,

phone: +90 5057958124, +90 2167730616 


\begin{tabular}{|l|l|l|l|l|l|}
\hline & $\begin{array}{l}\text { Faire une } \\
\text { analyse }\end{array}$ & $\varnothing$ & $\varnothing$ & $\varnothing$ & $\varnothing$ \\
\hline
\end{tabular}

Ce tableau montre que dans la première unité de ce manuel, les activités utilisent, dans leurs consignes, 5 verbes sur les 9 mentionnés au-dessus/dans notre longue introduction. Ce sont: " résumer », « réécrire », « rédiger », « formuler » et « regrouper ». Parmi les 8 activités qui nécessitent un acte de reformulation, 5 activités font appel aux compétences en expression/production écrite en partant de la compétence de la compréhension écrite. Dans ces activités les textes étendus (sources) sont choisis à partir de documents authentiques. Ces textes sont tirés de la presse comme « Le Point », "Courrier International », des extraits de la littérature française comme " Antigone » de Jean Anouilh, pièce écrite en 1942, des extraits de livres publiés sur des domaines aussi divers que " Le principe de Noé » de Michel Lacroix, Flammarion, 1997, « L'étudiant étranger » de Philippe Labro, Galimard 1986, Le psychologue, l'école et l'enfant de Bosetti, Dunodi 1995, des bandes dessinées «Agrippine et les inclus » de Claire Brétecher. La première phase demandée aux étudiants est, bien sûr, de comprendre le texte écrit représenté puis de le reformuler, de créer un texte reformulé par l'usage des différents processus linguistiques. Par exemple, il est à noter que le résumé semble être la méthode la plus utilisée lors de cette reformulation. Des activités telles que « En utilisant le vocabulaire ci-contre, résumez chaque texte en une phrase » ou « Résumez en une phrase... » demande aux apprenants la capacité de faire raccourcir un texte donné, c'est-à-dire, de pouvoir sélectionner les idées essentielles et d'éliminer les informations jugées secondaires ou redondantes. Cette activité se base sur la reformulation par la simplification. Une seule activité a été consacrée à la reformulation par la réécriture : "Reécrivez le dialogue en langue courante. » Cette activité visant à développer l'expression orale chez l'apprenant, nécessite la capacité de faire une transition entre les différents registres de la langue. Pouvoir faire une adaptation à un autre registre nécessite à la fois des prérequis linguistiques et un certain degré de créativité.

L'activité qui s'adresse à l'expression écrite chez l'apprenant pour la rédaction comporte la consigne suivante : "À partir des notes suivantes, rédigez deux paragraphes de trois phrases chacun. Dans le premier paragraphe, utilisez en priorité les constructions des rubriques 1 et 2 du tableau ci-contre. Dans le second, les constructions relatives. » Cette juxtaposition aboutit à un texte reformulé du texte étendu (deux notes présentées dans le manuel) par la complexification. L'activité qui demande aux apprenants de « Formuler l'idée principale développée par Michel Lacroix » part de la compréhension d'un texte étendu puis se finit par la reformulation de ce texte. Afin d'extraire l'idée principale d'un texte et de la transposer dans un texte reformulé, nécessite une compréhension puis élimination des informations secondaires ou auxiliaires.

Le développement de la compétence de l'expression orale est l'un des objectifs de l'apprentissage des langues étrangères. Dans cette unité, une seule activité s'adresse à cette compétence : « Faites un bref compte rendu de ces interventions. » Il s'agit d'une activité d'écoute. Après avoir écouté les interventions (le texte étendu), les apprenants devront résumer ce qu'ils ont entendu. Cette tâche demande une reformulation par simplification. Demander aux apprenants de faire des regroupements des éléments linguistiques ou des connaissances textuelles est une action souvent utilisée dans le processus d'apprentissage des langues étrangères.

Il y a 4 boîtes à outils dans cette unité. La reformulation par synonymie est utilisée dans toutes ces boîtes 4/4. D'autres sont la reformulation par explication 1/4, par définition 1/4, par dérivation 1/4 et par nominalisation 1/4. Dans cette unité, il existe 3 "préparation à l'écoute » qui se basent sur

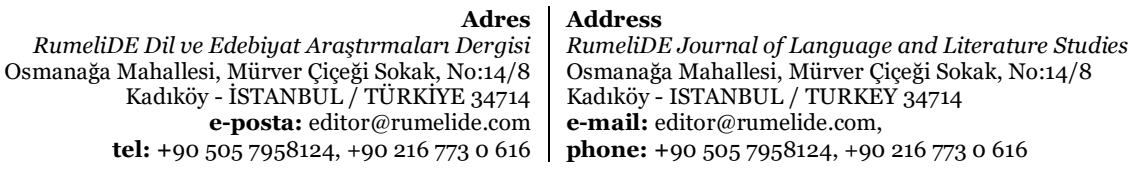


l'enseignement du « vocabulaire ». La reformulation par définition est utilisée 3/3, par synonymie 2/3, exemplification $1 / 3$, et par dérivation $1 / 3$.

\section{Panorama 4- Unité 2}

La deuxième unité qui est nommée « Récits » est divisée en 4 parties comme toutes les unités du manuel. Ces parties communes sont: « Analyses et Commentaires », "Dossier-Débat », «Simulation » et «Projet ». Cette deuxième unité se concentre sur les objectifs suivants : « Reformuler pour expliquer, généraliser, synthétiser. », "Mettre le contenu d'un texte en relation avec des connaissances et des opinions. », " Définir une notion. », « Passer des faits aux idées : poser un problème, généraliser. », «Passer des idées aux faits : illustrer, donner des exemples. », etc.

Tableau 2 : Unité 2

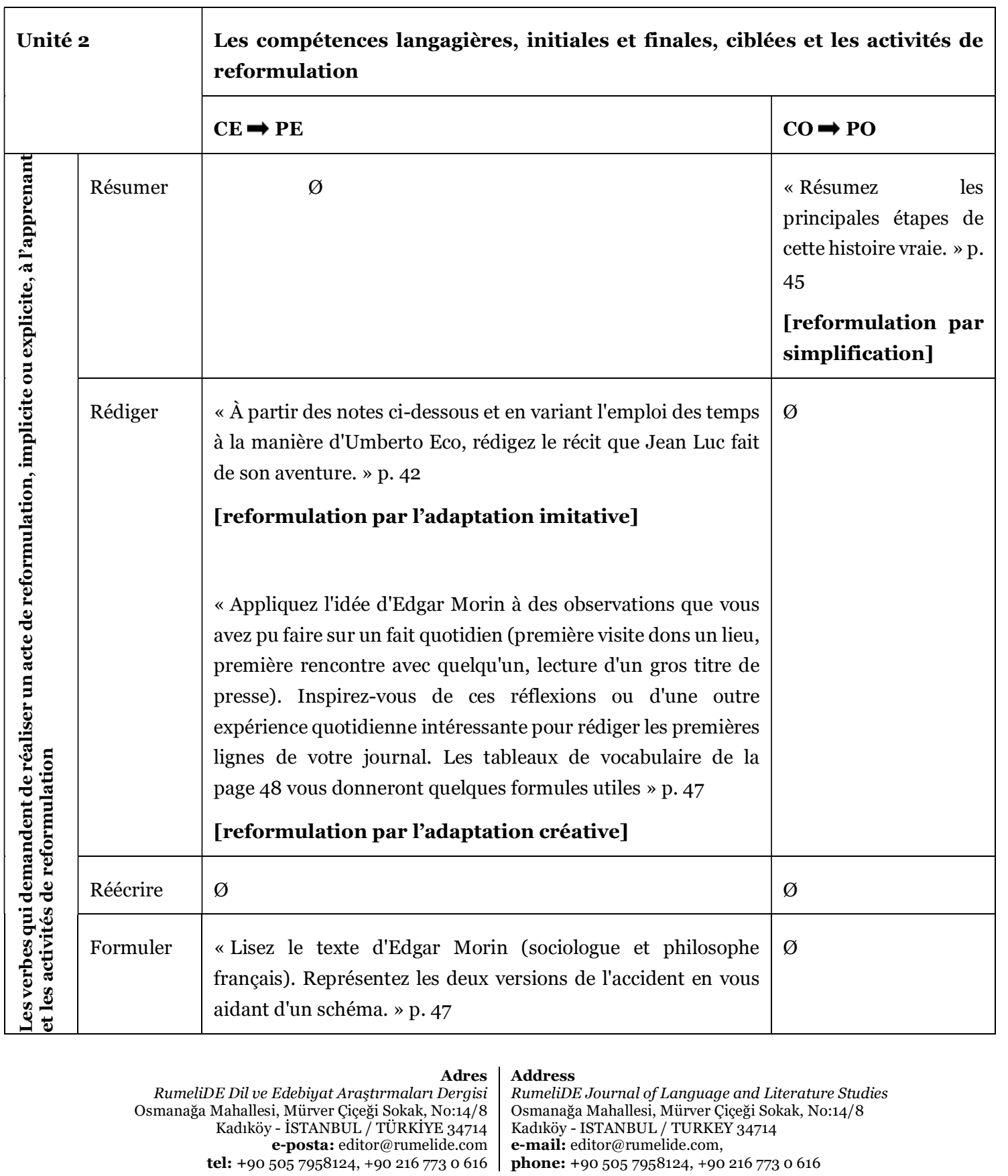


1014 / RumeliDE Journal of Language and Literature Studies 2021.24 (September)

Reformulation as a learning strategy for French as a foreign language: example from the "Panorama 4" manual / Ö. Sönmez (pp. 1004-1028)

\begin{tabular}{|c|c|c|}
\hline & reformulation par un replacement imitatif] & \\
\hline Reformuler & $\begin{array}{l}\text { «Reformulez le paragraphe en résumant les informations } \\
\text { essentielles d'une manière neutre. » } \mathrm{p} .31 \\
\text { reformulation par simplification] }\end{array}$ & $\varnothing$ \\
\hline Regrouper & $\varnothing$ & $\varnothing$ \\
\hline Définir & $\begin{array}{l}\text { «Lisez l'article ci-dessus. Définissez ce qu'est le bizutage. »p. } \\
35 \\
\text { reformulation par simplification] } \\
\text { «En vous appuyant sur ces définitions et sur vos } \\
\text { connaissances, complétez cet article de dictionnaire »p. } 38 \\
\text { reformulation par simplification] }\end{array}$ & $\varnothing$ \\
\hline $\begin{array}{l}\text { Faire une } \\
\text { synthèse }\end{array}$ & $\begin{array}{l}\text { «Faites une synthèse de ces opinions. » p. } 33 \\
\text { [reformulation par combinaison] }\end{array}$ & $\varnothing$ \\
\hline $\begin{array}{l}\text { Faire une } \\
\text { analyse }\end{array}$ & $\varnothing$ & $\varnothing$ \\
\hline
\end{tabular}

La $2^{\text {ème }}$ unité contient 22 activés. Parmi ces activités, 8 visent à développer l'acte de reformulation. Ces activités utilisent dans leurs consignes 6 verbes sur les 9 mentionnés. Ce sont : « résumer », " rédiger », « formuler », « reformuler », " définir » et « faire une synthèse ». Parmi ces 8 activités qui nécessitent un acte de reformulation, 7 activités s'adressent à l'expression/production écrite en partant de la compétence de la compréhension écrite. Dans ces activités les textes étendus (sources) sont choisis à partir de documents authentiques. Ces textes sont issus de presse tels que « Le Soir », «Libération », «Le monde », « Figaro », etc., de la littérature française comme « Les Confessions » de Jean-Jacques Rousseau, «Les Trois Mousquetaires » d'Alexandre Dumas, "Comment voyager avec un saumon» d'Umberto Eco, «Les Braban » de Patrick Bessons, « Pour sortir du XX ème siècle » d'Edgar Morin, etc. Il convient de noter ici que cette unité ne comprend pas d'activité de résumer visant à améliorer la compétence de la production écrite. D'autre part, on trouve l'activité suivante qui se focalise sur la compétence de la production orale en partant de la compétence de la compréhension orale : « Résumez les principales étapes de cette histoire vraie. » Il s'agit ici d'une activité d'écoute sur un récit d'une escroquerie. L'apprenant doit comprendre le document sonore et il doit éliminer les informations secondaires et reformuler un discours oral à la fois plus simplifié et intense.

L'activité qui nécessite un acte de reformulation par la rédaction est la suivante : «À partir des notes cidessous et en variant l'emploi des temps à la manière d'Umberto Eco, rédigez le récit que Jean-Luc fait de son aventure. » Cette activité demande aux apprenants de faire une reformulation par une adaptation imitative. Il a le texte d'Umberto Eco en tant que texte étendu et contient également les notes d'aventures de Jean-Luc. Quant à la deuxième activité d'écriture, "Appliquez l'idée d'Edgar Morin à des

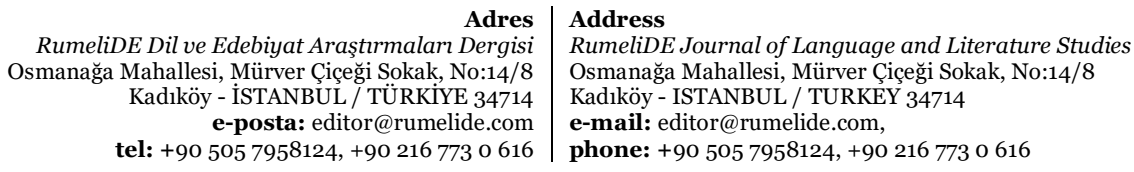


observations que vous avez pu faire sur un fait quotidien (première visite dans un lieu, première rencontre avec quelqu'un, lecture d'un titre de presse, ...). Inspirez-vous de ces réflexions ou d'une outre expérience quotidienne intéressante pour rédiger les premières lignes de votre journal. Les tableaux de vocabulaire de la page 48 vous donneront quelques formules utiles. », cette fois, l'apprenant est plus libre et créatif dans son acte de reformulation car ici il s'agit de l'expérience quotidienne ainsi que l'adaptation de l'idée d'Edgar Morin. " Lisez le texte d'Edgar Morin (sociologue et philosophe français). Représentez les deux versions de l'accident en vous aidant d'un schéma. » demande à l'apprenant une précision des savoirs sur l'accident et de les transférer sous une autre forme. Il s'agit ici une reformulation de remplacement imitatif. "Reformulez le paragraphe en résumant les informations essentielles d'une manière neutre. » vise à faire un résumé objectif de paragraphe en question. C'est une reformulation par la simplification de texte étendu. "Lisez l'article ci-dessus. Définissez ce qu'est le bizutage. », il s'agit aussi une simplification du texte étendu (l'article sur le bizutage). L'apprenant doit faire une définition au vu de ce qu'il a lu mais surtout compris. Cette définition sera le texte reformulé de l'article en question. Une autre activité qui a la consigne suivante, «En vous appuyant sur ces définitions et sur vos connaissances, complétez cet article de dictionnaire. » nécessite un acte de reformulation par la simplification des savoirs déjà présentés (sur l'adjectif "conservateur ») aux apprenants et faits une harmonisation avec leurs propres connaissances. La dernière activité de cette unité, " Faites une synthèse de ces opinions », s'agit de faire une synthèse deux documents authentiques de presse sur la protection de la nature. L'apprenant réalisera l'acte de reformulation par la combinaison des idées essentielles de deux articles (textes étendus) et de construire un texte reformulé par ces idées essentielles.

L'unité 2 représente 9 boîtes à outil. La reformulation par synonymie représente 8/9, par définition 1/9, la reformulation proprement dite est de $1 / 9$. L'unité 2 représente 6 "préparation à l'écoute ». La reformulation par définition est en proportion de $3 / 6$, par explication, $3 / 6$ et par synonymie $1 / 6$.

\section{Panorama 4- Unité 3}

La troisième unité nommée «Espaces » est, de même, divisée en 4 parties comme toutes les unités du manuel et porte les mêmes titres. Cette unité du manuel se concentre sur des objectifs comme: " Enchainer des idées et des arguments : arguments convergents (successions, liaisons) ; arguments divergents (opposition et concession). », « Nuancer, modérer, atténuer des opinions et des critiques. », " Etat des lieux et analyse des besoins (manques, aspirations, imaginaire collectif). », « Faire un compte rendu de séance orale. », etc.

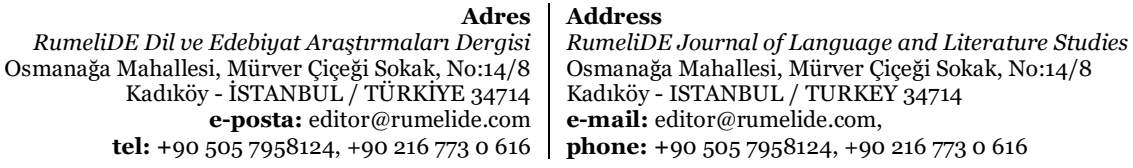

Adres
RumeliDE Dil ve Edebiyat Araştırmaları Dergisi Kahallesi, Mürver Çiçeği Sokak, No:14/8 el: +90 $5057958124,+902167730616$ 
1016 / RumeliDE Journal of Language and Literature Studies 2021.24 (September)

Reformulation as a learning strategy for French as a foreign language: example from the "Panorama 4" manual / Ö. Sönmez (pp. 1004-1028)

Tableau 3 : Unité 3

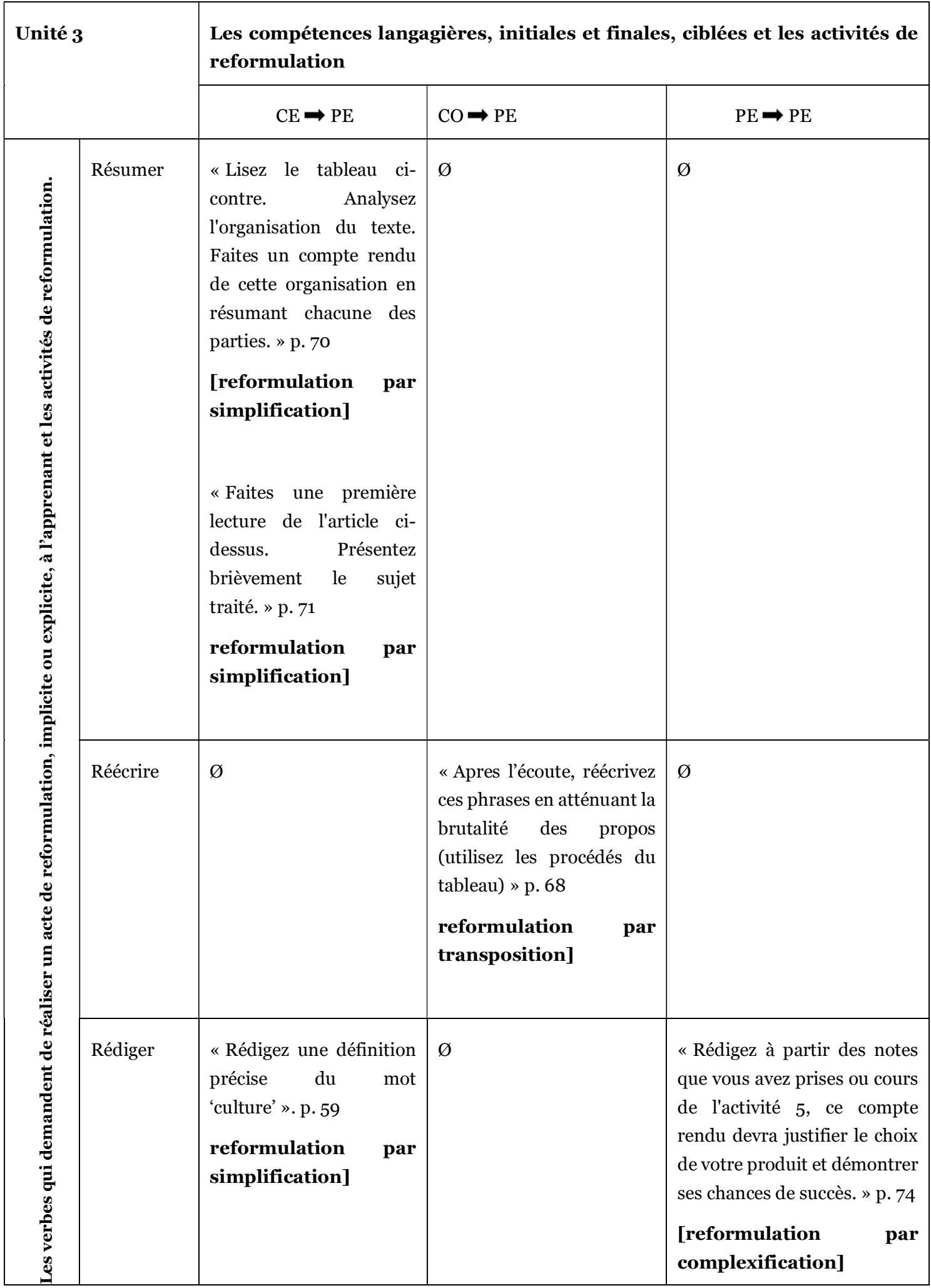

RumeliDE Dil ve Edebiyat Araştırmaları Dergisi Osmanağa Mahallesi, Mürver Çiçeği Sokak, No:14/8 Kadıköy - ISTANBUL / TÜRKIYE 34714 e-posta: editor@rumelide.com tel: +90 $5057958124,+902167730616$
Address

RumeliDE Journal of Language and Literature Studies Osmanağa Mahallesi, Mürver Çiçeği Sokak, No:14/8

Kadıköy - ISTANBUL / TURKEY 34714

e-mail: editor@rumelide.com

phone: +90 5057958124, +90 2167730616 


\begin{tabular}{|c|c|c|c|}
\hline Formuler & $\varnothing$ & $\varnothing$ & $\varnothing$ \\
\hline Reformuler & $\begin{array}{lr}\text { « Reformulez le texte } \\
\text { suivant sans employer les } \\
\text { mots qui appartiennent } \\
\text { ou vocabulaire de } \\
\text { l'espace. »p. } 57 \\
\text { reformulation par } \\
\text { simplification] }\end{array}$ & $\varnothing$ & $\varnothing$ \\
\hline Regrouper & $\varnothing$ & $\varnothing$ & $\varnothing$ \\
\hline Définir & $\begin{array}{l}\text { « Lisez le texte ci-dessus } \\
\text { et définissez brièvement } \\
\text { l'objectif de l'auteur. » } p \text {. } \\
55 \\
\text { [reformulation par } \\
\text { simplification] }\end{array}$ & $\begin{array}{l}\text { "Ecoutez le document. En } \\
\text { utilisant les formes } \\
\text { d'expression } \\
\text { l'opposition et de la } \\
\text { concession, donnez une } \\
\text { définition contrastée de } \\
\text { l'intégration et de } \\
\text { l'assimilation. "p. } 61 \\
\text { [reformulation par } \\
\text { simplification] }\end{array}$ & $\varnothing$ \\
\hline $\begin{array}{l}\text { Faire une } \\
\text { synthèse }\end{array}$ & $\begin{array}{l}\text { « Faites la synthèse des } \\
\text { constatations positives et } \\
\text { négatives de Théodore } \\
\text { Monod dans une note de } \\
15 \text { lignes. » p. } 68 \\
\text { [reformulation par } \\
\text { combinaison] }\end{array}$ & $\varnothing$ & $\varnothing$ \\
\hline $\begin{array}{l}\text { Faire une } \\
\text { analyse }\end{array}$ & $\varnothing$ & $\varnothing$ & $\varnothing$ \\
\hline
\end{tabular}

L'unité 3 contient 24 activités. Parmi ces 24 activités, 9 s'adressent à un acte de reformulation. Ces activités utilisent 6 verbes sur les 9 mentionnés. Ce sont : « résumer », « rédiger », " récrire », «reformuler », " définir » et «faire une synthèse ». Entre ces 9 activités, 6 visent à améliorer la compétence de production écrite en partant de la compétence de compréhension écrite. 2/9 prennent comme point de départ la compétence de compréhension orale et vise la production écrite. 1/9 est basé sur la compétence de production écrite et se focalise encore sur l'amélioration de la production écrite. Dans ces activités, les textes étendus (sources) sont choisis parmi des documents authentiques. Ces textes sont tirés de la presse comme Voyage Magazine, Le Monde, Le Figaro Magazine, Le Magazine de

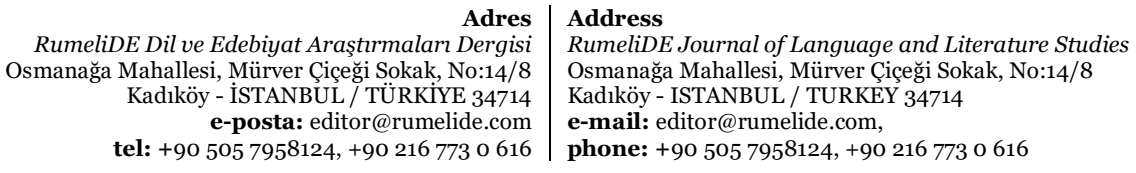


l'Express, Le Nouvel Observateur, le figaro économie, etc. et de la littérature française comme « Dans le labyrinthe » d'Alain Robbe-Grillet.

Dans cette unité, deux activités demandent aux apprenants de faire un résumé. La consigne de la première activité, « Lisez le tableau ci-contre. Analysez l'organisation du texte. Faites un compte rendu de cette organisation en résumant chacune des parties. » repose sur un travail de texte intitulé « Les nouveaux enjeux de la laïcité ». C'est le texte étendu pour l'apprenant. Le but est de résumer à l'aide d'un tableau qui représente certaines structures linguistiques liées à des sujets concernés. Cet acte de reformulation s'agit de la simplification du texte étendu. La deuxième activité, " Faites une première lecture de l'article ci-dessus. Présentez brièvement le sujet traité. », part comme la précédente de la compétence de compréhension écrite et nécessite d'éliminer les informations secondaires du texte étendu afin de pouvoir le reformuler. La consigne de l'activité suivante, "Rédigez une définition précise du mot " culture " » et « Lisez le texte ci-dessus et définissez brièvement l'objectif de l'auteur » s'agissent d'une reformulation par simplification des textes donnés sur la culture et nécessitent une capacité à pouvoir choisir les particularités essentielles d'une notion. " Reformulez le texte suivant sans employer les mots qui appartiennent ou vocabulaire de l'espace. » est un autre type d'activité qui se base sur la simplification de texte étendu en éliminant les vocabulaires de l'espace. Quant à l'activité «Faites la synthèse des constatations positives et négatives de Théodore Monod dans une note de 15 lignes. », celleci s'agit d'une reformulation par combinaison des constatations positives et négatives de texte étendu.

Cette unité comprend 2 activités qui partent de la compétence de la compréhension orale et qui demandent aux apprenants de faire une production écrite. La première activité consiste en un acte de reformulation par simplification: "Après l'activité d'écoute, réécrivez ces phrases en atténuant la brutalité des propos (utilisez les procédés du tableau) ». Cette simplification implique la reproduction d'un texte moins «brutal» que le texte étendu. L'autre activité qui a comme consigne «Ecoutez le document. En utilisant les formes d'expression de l'opposition et de la concession, donnez une définition contrastée de l'intégration et de l'assimilation. » Il s'agit d'un acte de reformulation de simplification de document oral. Cette tâche nécessite la capacité de pouvoir choisir les particularités essentielles des notions et de pouvoir les comparer. La dernière activité est basée sur une autre activité de production écrite déjà accomplie par l'apprenant. "Rédigez à partir des notes que vous avez prises ou cours de l'activité 5 , ce compte rendu devra justifier le choix de votre produit et démontrer ses chances de succès ». Ces notes déjà préparées par l'apprenant sont des textes étendus. Il s'agit d'un acte de reformulation par la complexification, car l'apprenant doit faire des justifications en reformulant le texte étendu.

Dans cette unité, 7 boîtes à outils contiennent la reformulation par synonymie. Une seule boîte contient la reformulation par explication et synonymie. On trouve 2 « préparation à l'écoute ». L'une se base sur la reformulation par définition et explication, l'autre, sur la synonymie.

\section{Panorama 4- Unité 4}

La quatrième unité intitulée "Interrogations » divisée aussi en 4 parties portant les mêmes titres, se concentre sur les objectifs suivants : « Faire une démonstration (induire, déduire, faire des hypothèses, confronter des faits). », " Négocier : de l'accord à la réticence ; de la réticence au désaccord », " La cohérence du récit. », « Figures de la condition (implications, dépendances, restrictions, réserves). », etc.

\footnotetext{
Adres

\begin{tabular}{r|l} 
Adres & Address \\
RumeliDE Dil ve Edebiyat Araştırmaları Dergisi & RumeliDE Journal of Language and Literature Studies
\end{tabular} Osmanağa Mahallesi, Mürver Çiçeği Sokak, No:14/8 $\quad$ Osmanağa Mahallesi, Mürver Çiçeği Sokak, No:14/8 Kadıköy - ÍSTANBUL / TÜRKIYE 34714 Kadıköy - ISTANBUL / TURKEY 34714 e-posta: editor@rumelide.com e-mail: editor@rumelide.com, tel: +90 505 7958124, +90 2167730616 phone: +90 505 7958124, +90 2167730616
} 
Tableau 4 : Unité 4

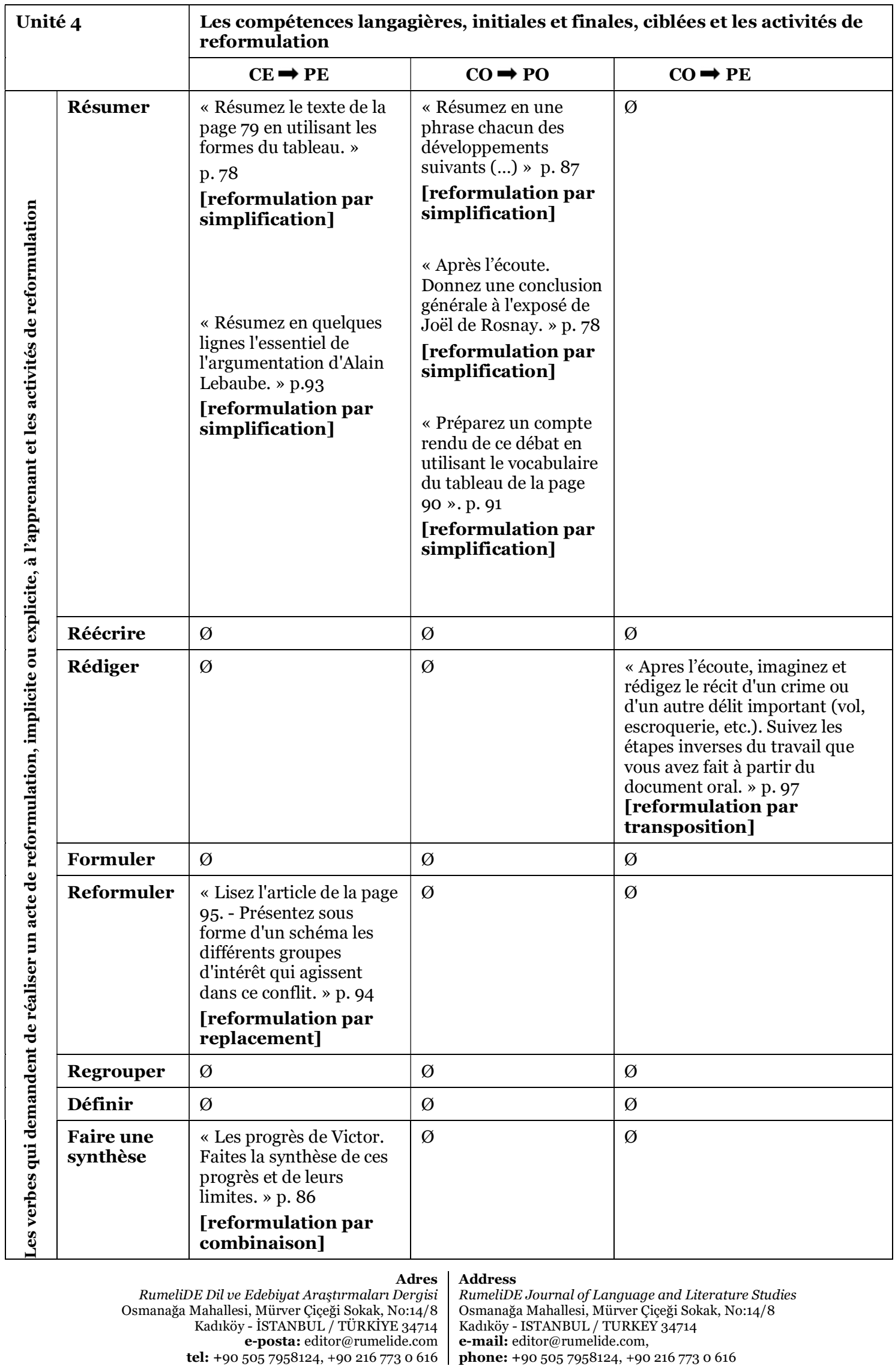




\begin{tabular}{|l|l|l|l|l|}
\hline $\begin{array}{l}\text { Faire une } \\
\text { analyse }\end{array}$ & $\varnothing$ & $\varnothing$ & $\varnothing$ \\
\hline
\end{tabular}

L'unité 4 contient 22 activités. De ces 22 activités, 8 font appel à un acte de reformulation. Ces activités utilisent 4 des 9 verbes mentionnés. Ce sont : « résumer », « rédiger », "reformuler », et « faire une synthèse ». Parmi ces 8 activités, 4 visent à améliorer la compétence de production écrite en partant de la compétence de compréhension écrite, $3 / 8$ visent à améliorer la compétence de production orale en partant de la compétence de compréhension orale. $1 / 8$ vise à améliorer la compétence de production écrite en partant de la compétence de compréhension orale. Dans ces activités les textes étendus (sources) sont choisis à partir de documents authentiques. Ces textes sont issus de presse tels que Courrier International, Le Point, etc. et de la littérature française comme « La guerre de Troie n'aura pas lieu » de Jean Giraudoux, "Du fer dans les épinards et d'autres idées reçues », des livres sur divers domaines comme «Le Roman policier» d'André Vanoncini, «Les enfants Sauvages » de Lucien Malson, etc.

Dans cette unité les apprenants doivent résumer 2 activités sur 8. La première activité, « Résumez le texte de la page 79 en utilisant les formes du tableau ", est une reformulation en simplifiant le texte étendu et en imitant la structure linguistique fournie dans la boîte à outils. Deuxième activité, «Résumez en quelques lignes l'essentiel de l'argumentation d'Alain Lebaube» consiste à la reformulation par simplification de texte étendu d'Alain Lebaube.

«Lisez l'article de la page 95. - Présentez sous forme d'un schéma les différents groupes d'intérêt qui agissent dans ce conflit. » Il s'agit d'un acte de reformulation par un replacement des savoirs du texte étendu et de le reconstruire sous une autre forme. "Les progrès de Victor. Faites la synthèse de ces progrès et de leurs limites. » demande à l'apprenant de faire la synthèse de l'article donné. Il s'agit d'un acte de reformulation par la combinaison des informations du texte étendu.

3 activités, qui visent à améliorer la compétence de compréhension/production orale demandent aux apprenants de faire des résumés de documents audios. Toutes ces activités requièrent un acte de reformulation par simplification des textes étendus. Par contre, la dernière activité de ces trois demandes l'utilisation des vocabulaires donnés dans le tableau attaché à l'exercice. La seule activité qui a comme consigne: « Après l'écoute, imaginez et rédigez le récit d'un crime ou d'un autre délit important (vol, escroquerie, etc.). Suivez les étapes inverses du travail que vous avez fait à partir du document oral. » nécessite un acte de reformulation par transposition. Dans cette unité, 2 boîtes à outils et 3 « préparation à l'écoute » contiennent la reformulation par synonymie.

\section{Panorama 4- Unité 5}

La cinquième unité qui est nommée «Passions » est divisée en 4 parties comme toutes les unités du manuel. Cette cinquième unité se concentre sur les objectifs suivants : "Expression des sensations et des émotions. ", "Comparaisons, identifications, métaphores. ", "Jeux de mots et calembours. ", "Commenter une scène de roman.", "Comparer une œuvre romanesque et son adaptation cinématographique. », etc.

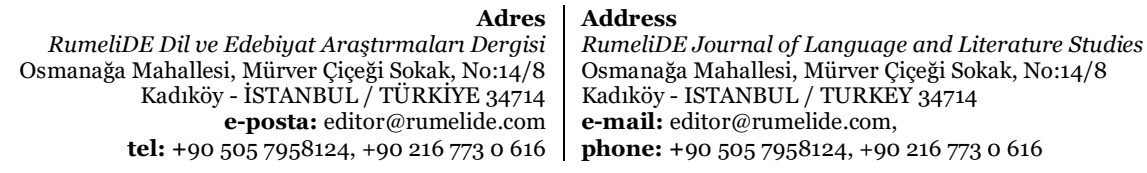


Tableau 5 : Unité 5

\begin{tabular}{|c|c|c|c|c|c|}
\hline \multirow{2}{*}{\multicolumn{2}{|c|}{ Unité 5}} & \multicolumn{4}{|c|}{$\begin{array}{l}\text { Les compétences langagières, initiales et finales, ciblées et les activités de } \\
\text { reformulation }\end{array}$} \\
\hline & & $\mathrm{CE} \rightarrow \mathrm{PE}$ & $\mathrm{CO} \rightarrow \mathrm{PH}$ & $\mathrm{CO} \rightarrow \mathrm{PO}$ & $\mathrm{CE}+\mathrm{CO} \rightarrow \mathrm{PE}$ \\
\hline \multirow{2}{*}{ 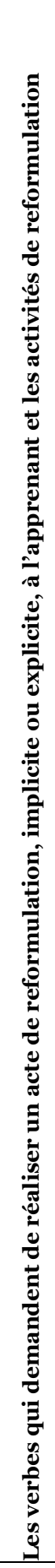 } & Résumer & $\begin{array}{l}\text { «'Résumez ce que } \\
\text { l'auteur a voulu } \\
\text { exprimer. » p. 101 } \\
\text { [reformulation } \\
\text { par } \\
\text { simplification] } \\
\text { « Résumez l'essentiel } \\
\text { de l'article. » p. 103 } \\
\text { [reformulation } \\
\text { par } \\
\text { simplification] } \\
\text { « Résumez le récit ci- } \\
\text { dessus en utilisant les } \\
\text { mots suivants : p. } \\
\text { 103 } \\
\text { [reformulation } \\
\text { par } \\
\text { simplification] } \\
\text { « Vous êtes } \\
\text { documentaliste ou } \\
\text { Nouvel Observateur. } \\
\text { Pour les archives du } \\
\text { journal, rédigez un } \\
\text { résumé de cet article } \\
\text { en 10 lignes ».p.103 } \\
\text { [reformulation } \\
\text { par } \\
\text { simplification] } \\
\text { « Donnez une } \\
\text { interprétation } \\
\text { générale du conte. » } \\
\text { p. 129 } \\
\text { [reformulation } \\
\text { par } \\
\text { simplification] }\end{array}$ & $\varnothing$ & $\varnothing$ & $\varnothing$ \\
\hline & Réécrire & $\begin{array}{l}\text { "Réécrivez deux fois } \\
\text { le texte ci-dessus en } \\
\text { remplaçant les mots } \\
\text { en gras par les mots } \\
\text { ci-dessus. Dans votre } \\
\text { première réécriture, } \\
\text { vous puiserez vos } \\
\text { métaphores dans le } \\
\text { vocabulaire de } \\
\text { l'agriculture. Dans } \\
\text { votre deuxième } \\
\text { réécriture, vous }\end{array}$ & $\varnothing$ & $\varnothing$ & $\varnothing$ \\
\hline & $\begin{array}{r}\text { Rur } \\
\text { Osmar }\end{array}$ & $\begin{array}{l}\text { E Dil ve Edebiyat Araşttrmalc } \\
\text { Mahallesi, Mürrer Çiçeği Sokkal } \\
\text { Kadıköy - İSTANBUL / TÜRK } \\
\text { e-posta: editor@ @rum } \\
\text { el: +90 505 7958124, +90 } 216\end{array}$ & \begin{tabular}{r|l} 
Adres & A \\
Dergisi & $R$ \\
No:14/8 & $\mathrm{O}$ \\
YE 34714 & $\mathrm{~K}$ \\
lide.com & $\mathbf{e}$ \\
773 o 616 & $\mathbf{p}$
\end{tabular} & $\begin{array}{l}\text { Irnal of Langua } \\
\text { hallesi, Mürver } \\
\text { ANBUL / TURK } \\
\text { r@ rumelide.com } \\
5057958124,+9\end{array}$ & $\begin{array}{l}\text { Ire Studies } \\
\text { Jo:14/8 }\end{array}$ \\
\hline
\end{tabular}




\begin{tabular}{|c|c|c|c|c|c|}
\hline & $\begin{array}{l}\text { utiliserez le } \\
\text { vocabulaire } \\
\text { militaire. " p. } 105 \\
\text { [reformulation } \\
\text { par } \\
\text { complexification] }\end{array}$ & & & & \\
\hline Rédiger & $\begin{array}{l}\text { «Lisez la lettre } \\
\text { d'Elia. En utilisant le } \\
\text { vocabulaire ci-contre, } \\
\text { rédigez la première } \\
\text { partie de votre } \\
\text { lettre. » p. } 114 \\
\text { [reformulation } \\
\text { par imitation] }\end{array}$ & $\varnothing$ & & $\varnothing$ & $\begin{array}{l}\text { «Imaginez et } \\
\text { rédigez ce script } \\
\text { de la scène } \\
\text { filmée. Comme } \\
\text { dans celui de la } \\
\text { scène du Rouge } \\
\text { et le Noir. " p. } \\
123 \\
\text { [reformulation } \\
\text { par } \\
\text { transposition } \\
\text { et adaptation] }\end{array}$ \\
\hline Formuler & $\begin{array}{l}\text { «Lisez le texte de la } \\
\text { page } 101 \text {. Formulez } \\
\text { de façon banale ce } \\
\text { que raconte Philippe } \\
\text { Delerm. » p. } 101 \\
\text { [reformulation } \\
\text { par adaptation à } \\
\text { un autre registre] }\end{array}$ & $\varnothing$ & & $\varnothing$ & $\varnothing$ \\
\hline Reformuler & $\begin{array}{l}\text { « Reformulez Ia } \\
\text { phrase d'une manière } \\
\text { plus banale. » p. } 104 \\
\text { [reformulation } \\
\text { par adaptation à } \\
\text { un autre registre] } \\
\text { « En utilisant les } \\
\text { constructions } \\
\text { données dans le } \\
\text { tableau reformulez la } \\
\text { lettre ci-dessous de } \\
\text { façon que les } \\
\text { sentiments indiqués } \\
\text { en marge soient } \\
\text { exprimés. » p. 113 } \\
\text { [reformulation } \\
\text { par replacement] }\end{array}$ & $\varnothing$ & & $\varnothing$ & $\varnothing$ \\
\hline Regrouper & $\varnothing$ & $\varnothing$ & & $\varnothing$ & $\varnothing$ \\
\hline Définir & $\varnothing$ & $\begin{array}{l}\text { «À par } \\
\text { l'intervi } \\
\text { dessus, } \\
\text { définire } \\
\text { caracté } \\
\text { de la no } \\
\text { spiritua } \\
\text { parle Fr } \\
\text { Champ } \\
127\end{array}$ & $\begin{array}{l}\text { ir de } \\
\text { ew ci- } \\
\text { jous } \\
\text { les } \\
\text { istiques } \\
\text { uvelle } \\
\text { ité dont } \\
\text { ançoise } \\
\text { on p. }\end{array}$ & $\begin{array}{l}\text { «À partir de } \\
\text { l'interview ci-dessus, } \\
\text { vous définirez les } \\
\text { caractéristiques de la } \\
\text { nouvelle spiritualité } \\
\text { dont parle Françoise } \\
\text { Champion » p. } 127 \\
\text { [reformulation } \\
\text { par } \\
\text { simplification] }\end{array}$ & $\varnothing$ \\
\hline \multicolumn{3}{|c|}{$\begin{array}{r}\text { Adres } \\
\text { RumeliDE Dil ve Edebiyat Araştrmaları Dergisi } \\
\text { Osmanağa Mahallesi, Mürver Ççeği Sokak, No:14/8 } \\
\text { Kadıköy - İTANBUL / TÜRKIYE } 34714 \\
\text { e-posta: editor@rumelide.com } \\
\text { tel: +90 505 7958124, +90 } 2167730616\end{array}$} & $\begin{array}{l}\text { Address } \\
\text { RumeliDE } \\
\text { Osmanağa } \\
\text { Kadıköy- } \\
\text { e-mail: e } \\
\text { phone: + }\end{array}$ & $\begin{array}{l}\text { Irnal of Language and Liter } \\
\text { hallesi, Mürver Çiçeği Sokak, } \\
\text { ANBUL / TURKEY } 34714 \\
\text { r@rumelide.com, } \\
\text { 505 7958124, +90 } 216773 \text { o } 6\end{array}$ & $\begin{array}{l}\text { re Studies } \\
0: 14 / 8\end{array}$ \\
\hline
\end{tabular}




\begin{tabular}{|c|c|c|c|c|}
\hline & & $\begin{array}{l}\text { [reformulation } \\
\text { par } \\
\text { simplification] }\end{array}$ & & \\
\hline $\begin{array}{l}\text { Faire une } \\
\text { synthèse }\end{array}$ & $\varnothing$ & $\varnothing$ & $\varnothing$ & $\varnothing$ \\
\hline $\begin{array}{l}\text { Faire une } \\
\text { analyse }\end{array}$ & $\begin{array}{l}\text { «En vous appuyant } \\
\text { sur les documents de } \\
\text { cette double page et } \\
\text { sur vos propres } \\
\text { opinions, vous } \\
\text { analyserez le goût } \\
\text { actuel pour les } \\
\text { aventures extrêmes. } \\
\text { Vous rechercherez } \\
\text { plus particulièrement } \\
\text { les motivations pour } \\
\text { ce type d'activités à } \\
\text { risque. Votre exposé } \\
\text { oral ou écrit devra } \\
\text { inclure des citations } \\
\text { directes ou indirectes } \\
\text { des documents (voir } \\
\text { texte de la page 109 } \\
\text { et tableau de la page } \\
\text { 111). » p. 110 } \\
\text { [reformulation } \\
\text { par } \\
\text { complexification] }\end{array}$ & $\varnothing$ & $\begin{array}{l}\text { En vous appuyant sur } \\
\text { les documents de } \\
\text { cette double page et } \\
\text { sur vos propres } \\
\text { opinions, vous } \\
\text { analyserez le goût } \\
\text { actuel pour les } \\
\text { aventures extrêmes. } \\
\text { Vous rechercherez } \\
\text { plus particulièrement } \\
\text { les motivations pour } \\
\text { ce type d'activités à } \\
\text { risque. Votre exposé } \\
\text { oral ou écrit devra } \\
\text { inclure des citations } \\
\text { directes ou indirectes } \\
\text { des documents (voir } \\
\text { texte de la page 109 } \\
\text { et tableau de la page } \\
\text { 111). p. 110 } \\
\text { [reformulation } \\
\text { par } \\
\text { complexification] }\end{array}$ & $\varnothing$ \\
\hline
\end{tabular}

L’unité 5 contient 19 activités. Sur ces 19 activités, 13 nécessitent un acte de reformulation. Ces activités utilisent 7 des 9 verbes mentionnés au-dessus dans leurs consignes. Ce sont : « résumer », « réécrire » », « rédiger », « formuler », « reformuler », « définir », et « faire une analyse ». Parmi ces 13 activités, 11 visent à améliorer la compétence de production écrite en partant de la compétence de compréhension orale. Une activité entre ces 11 peut également être acceptée dans le groupe d'activités visant à améliorer la compétence de production orale en partant de la compétence de compréhension orale, car la consigne implique les deux. Une seule activité s'adresse à la fois aux compétences de production écrite et orale en partant de la compréhension orale, car sa consigne n'est pas précise. 2/13 visent à améliorer la compétence de production écrite en partant de la compétence de compréhension orale. La dernière activité combine deux compétences (la compréhension écrite et orale) et l'analyse d'une photo en visant la production écrite. Dans ces activités les textes étendus (sources) sont choisis à partir de documents authentiques. Ces textes sont tirés de la presse comme Le Nouvel Observateur, Le monde, Le point, etc. et de la littérature française comme «Les Mots » de Jean-Paul Sartre, «Correspondance » d'Albert Camus, « Le Rouge et le Noir » de Stendhal, « La séquence de la soirée sous le tilleul dans le téléfilm Le Rouge et le Noir. Adaptation : Danièle Thompson et Jean-Daniel Verhaeghe », « Madame Bovary » de Gustave Flaubert, etc.

Dans cette unité, 5 activités sur 13 demandent aux apprenants de faire des résumés. Toutes ces activités sont basées sur la compétence de compréhension écrite et visent à améliorer la compétence de la production écrite chez l'apprenant. Parmi ces 5 activités de résumé, 3 activités nécessitent un acte de reformulation par simplification des textes étendus qui repose sur la généralisation des idées essentielles et l'élimination des informations secondaires. Les deux autres activités s'adressent à la fois à un acte de reformulation par simplification mais aussi à l'utilisation des nouveaux vocabulaires donnés ("Résumez le récit ci-dessus en utilisant les mots suivants : (...)») et de faire une simulation en assumant un rôle

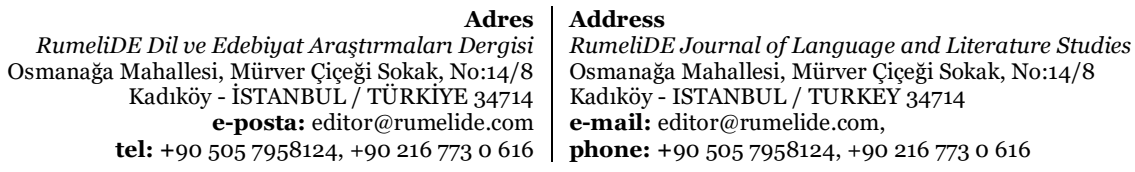


fictif ( «Vous êtes documentaliste au Nouvel Observateur. Pour les archives du journal, rédigez un résumé de cet article en 10 lignes. »). L'activité qui a la consigne : « Réécrivez deux fois le texte ci-dessus en remplaçant les mots en gras par les mots ci-dessus. Dans votre première réécriture, vous puiserez vos métaphores dans le vocabulaire de l'agriculture. Dans votre deuxième réécriture, vous utiliserez le vocabulaire militaire. » Il s'agit d'un acte de reformulation par la complexification car l'apprenant doit reformuler le texte étendu par les mots en gras et utiliser des nouveaux mots. L'activité suivante, « Lisez la lettre d'Elia. En utilisant le vocabulaire ci-contre, rédigez la première partie de votre lettre. », s'appuie sur l'acte de reformulation par l'imitation du texte étendu mais avec la contrainte supplémentaire: utilisation d'un nouveau vocabulaire dans le texte reformulé. Cette unité nécessite de la part des apprenants deux activités distinctes : la reformulation et aussi l'adaptation du texte à un autre registre de la langue.

«En utilisant les constructions données dans le tableau reformulez la lettre ci-dessous de façon que les sentiments indiqués en marge soient exprimés » s'agit d'un acte de reformulation par replacement des savoirs déjà donnés dans la lettre (le texte étendu) en impliquant les structures linguistiques données dans le tableau. Cette activité comporte la consigne suivante : « En vous appuyant sur les documents de cette double page et sur vos propres opinions, vous analyserez le goût actuel pour les aventures extrêmes. Vous rechercherez plus particulièrement les motivations pour ce type d'activités à risque. Votre exposé oral ou écrit devra inclure des citations directes ou indirectes des documents (voir texte de la page 109 et tableau de la page 111). » Elle repose sur une analyse des documents écrits donnés mais fait aussi intervenir les opinions personnelles des apprenants. Il s'agit d'un acte de reformulation par complexification, car les apprenants doivent également inclure des citations directes ou indirectes des documents. L'activité qui se base sur un document audio a la consigne suivante : «À partir de l'interview ci-dessus, vous définirez les caractéristiques de la nouvelle spiritualité dont parle Françoise Champion ». Il s'agit d'un acte de reformulation par la simplification du texte étendu (l'interview).

La dernière activité de cette unité est «Imaginez et rédigez ce script de la scène filmée. Comme dans celui de la scène du Rouge et le Noir. ». Il s'agit d'un acte de reformulation par la transposition et par l'adaptation du texte étendu.

Cette unité contient 7 boîtes à outils. Toutes se basent à la reformulation par synonymie. Il existe 2 «préparation à l'écoute ». L'une se base à la reformulation par explication, définition et synonymie conjointement. L'autre comporte la reformulation par synonymie et définition.

\section{Panorama 4- Unité 6}

La sixième unité nommée « Valeurs » divisée en 4 parties et intitulée de même comme toutes les unités du manuel, se concentre sur les objectifs suivants : "Commenter des statistiques et des sondages. », " Exprimer l'importance ou la banalité. », « Atténuer une information ou l'exagérer. », " Argumenter un projet. », etc.

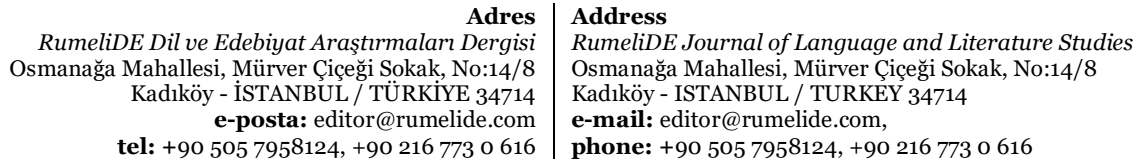


Tableau 6 : Unité 6

\begin{tabular}{|c|c|c|}
\hline \multicolumn{2}{|c|}{ Unité 6} & \multirow{2}{*}{$\begin{array}{l}\text { Les compétences langagières, initiales et finales, ciblées et les activités de } \\
\text { reformulation } \\
\qquad \mathrm{CE} \rightarrow \mathrm{PE}\end{array}$} \\
\hline \multirow{10}{*}{$\begin{array}{l} \\
\end{array}$} & & \\
\hline & Résumer & $\begin{array}{l}\text { "Répartissez-vous les trois parties de ce dossier. Chaque groupe préparera un } \\
\text { compte rendu des résultats des sondages et des exemples présentés dans la partie } \\
\text { dont il s'occupe. (Utilisez le vocabulaire du tableau de la page 133.) » p. } 130 \\
\text { [reformulation par complexification] }\end{array}$ \\
\hline & Réécrire & $\varnothing$ \\
\hline & Rédiger & $\varnothing$ \\
\hline & Formuler & $\begin{array}{l}\text { "Lisez ci-contre la brève nouvelle communiquée par la préfecture de Nîmes à la } \\
\text { presse un lundi à } 18 \text { heures. Imaginez quatre formulations de plus en plus } \\
\text { dramatiques de cette nouvelle. » p. } 140 \\
\text { [reformulation par complexification] }\end{array}$ \\
\hline & Reformuler & $\begin{array}{l}\text { «Pour chaque paragraphe, indiquez l'objectif de l'auteur. Reformulez brièvement } \\
\text { l'argument développé et son éventuel commentaire. » p. } 145 \\
\text { [reformulation par complexification] }\end{array}$ \\
\hline & Regrouper & $\varnothing$ \\
\hline & Définir & $\varnothing$ \\
\hline & $\begin{array}{l}\text { Faire une } \\
\text { synthèse }\end{array}$ & $\varnothing$ \\
\hline & $\begin{array}{l}\text { Faire une } \\
\text { analyse }\end{array}$ & $\varnothing$ \\
\hline
\end{tabular}

L'unité 6 contient 19 activités. Sur ces 19 activités seulement 3 s'adressent à un acte de reformulation. Ces activités utilisent 3 des 9 verbes mentionnés au-dessus dans leurs consignes. Ce sont : "résumer », « formuler » et « reformuler ». Toutes ces 3 activités visent à améliorer la compétence de production écrite en partant de la compétence de compréhension écrite. Dans ces activités, les textes étendus (sources) sont sélectionnés à partir de documents authentiques. Ces textes sont issus de presse tels que Le monde, Le point, etc., de la littérature française comme « Candide ou I'optimiste » et de la sociologie comme « Sur la télévision » de Pierre Bourdieu.

La première activité comporte les instructions suivantes : "Répartissez-vous les trois parties de ce dossier. Chaque groupe préparera un compte rendu des résultats des sondages et des exemples présentés dans la partie dont il s'occupe. (Utilisez le vocabulaire du tableau de la page 133.) » Cette activité est basée sur des articles et des sondages comme texte étendu. Les apprenants doivent faire un acte de reformulation par complexification, car le compte rendu doit impliquer le nouveau vocabulaire appris. La deuxième activité consiste en un acte de reformulation par complexification. Elle a la consigne suivante : «Lisez ci-contre la brève nouvelle communiquée par la préfecture de Nîmes à la presse un lundi à 18 heures. Imaginez quatre formulations de plus en plus dramatiques de cette nouvelle.» L'apprenant doit s'appuyer sur la communiquée comme texte étendu et il doit en reformuler quatre textes qui exagèrent la situation donnée. La dernière activité se base sur un article intitulé « L'ambition Francophone » tiré du journal « Le Monde ». Sa consigne est la suivante : "Pour chaque paragraphe, indiquez l'objectif de l'auteur. Reformulez brièvement l'argument développé et son éventuel commentaire. » Il s'agit d'un acte de reformulation par simplification, car l'apprenant doit déterminer les idées essentielles de l'auteur en éliminant les informations secondaires.

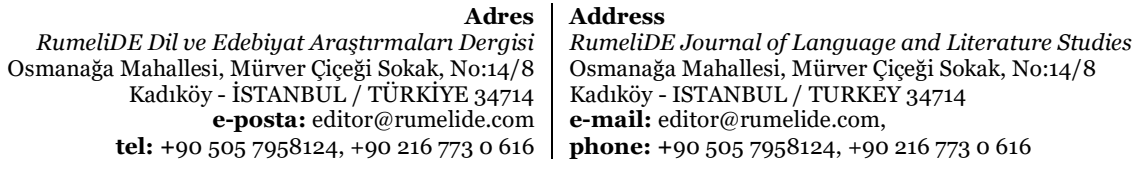


Cette unité contient 5 boîtes à outils. Toutes se basent à la reformulation par synonymie. Il existe 5 «préparation à l'écoute». 3 se basent sur la reformulation par définition et 2 autres sur explication.

\section{Conclusion}

Dans cette recherche, nous avons essayé d'examiner comment « l'acte de reformulation », qui peut être considéré comme une créativité linguistique, et qui est un sujet de recherche dans de nombreux domaines scientifiques tels que la linguistique, l'enseignement des langues étrangères, la traduction, est utilisé dans le manuel français Panorama 4, niveau B2. Ce manuel, rédigé selon les principes de la méthode communicative, comprend 6 unités et un total de 132 activités. Dans la classification que nous avons réalisée dans notre étude, nous avons constaté que la reformulation a été utilisée explicitement ou implicitement dans 47 de ces activités. Dans 33 de ces 47 activités, c'est premièrement la compétence de compréhension écrite qui est attendue de l'apprenant. Au terme de ces 33 activités basées sur la compétence de compréhension écrite, l'apprenant est invité à faire une production écrite en réalisant un acte de reformulation. Dans 7 de ces activités, on s'attend principalement à ce que l'apprenant ait une meilleure compétence de la compréhension orale. À la fin de ces activités, l'apprenant est censé suffisamment maitriser l'expression orale pour réaliser un acte de reformulation. Dans 3 des activités, l'apprenant commence par la compréhension orale et à la fin de ces activités, on attend de lui qu'il réalise une expression écrite par la reformulation. Dans 2 des activités, l'apprenant est censé faire une communication écrite à partir d'une expression écrite déjà réalisée et enfin, dans 2 autres activités, l'apprenant doit combiner, au moins, deux des compétences linguistiques (compréhension écrite et orale) et faire une expression écrite par le biais de la reformulation. Dans la consigne de 47 activités, le verbe " résumer » a été utilisé 15 fois, « rédiger » 8 , « reformuler » 6 , « formuler » 5 , « définir » 5 , " réécrire » 4, « faire une synthèse » 3 et " faire une analyse » 1 seule fois. Sur les 47 activités que l'apprenant est censé accomplir et procéder à un acte de reformulation, 25 d'entre elles sont réalisés par simplification, 6 par complexification, 5 par replacement, encore 5 par adaptation, 3 par transposition, 2 par combinaison et 1 par imitation. Un total de 34 boîtes d'aide est utilisé dans les 6 unités. La reformulation, l'est, dans la quasi-totalité de ces boîtes d'aide, qui reflètent les usages linguistiques, les structures de grammaire et de syntaxe destinées à être enseignés à l'apprenant dans l'unité concernée. La synonymie, n'est présente que dans, seulement, 3 boîtes. Explication, définition, nominalisation, dérivation et exemplification ont également été utilisées avec la synonymie. Dans les 6 unités, un total de 21 boîtes "préparation à l'écoute » incluant l'enseignement du vocabulaire a été utilisé. Dans 18 de ces 21 boîtes, la reformulation est effectuée par définition.

L'analyse des résultats de notre recherche portant sur le manuel Panorama 4, basé sur l'approche communicative et pour le niveau B2, nous montre que :

*36\% de toutes les activités proposées à l'apprenant dans le manuel incluent un acte de reformulation

* $71 \%$ des activités proposées dans le manuel et impliquant un « acte de reformulation » partent de la compétence écrite et visent à la production écrite

* $15 \%$ des activités décrites dans le manuel et impliquant un "acte de reformulation » partent de la compétence écrite et ciblent la production écrite

*6\% des activités présentées dans le manuel et impliquant un " acte de reformulation » partent de la compétence orale et sont destinées à la production écrite

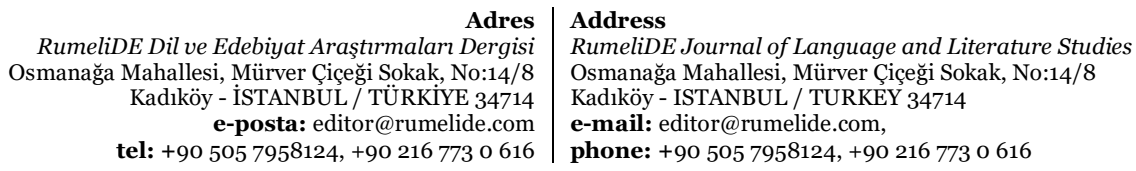


*4\% des activités mentionnées dans le manuel et impliquant un « acte de reformulation » partent d'une expression écrite déjà faite par l'apprenant et ciblent une autre production écrite

*4\% des activités présentées dans le manuel et impliquant un « acte de reformulation » partent de la combinaison des compétences écrites et orales, et visent à la production écrite

*Dans la consigne de 47 activités, le verbe « résumer » a été utilisé $32 \%$, « rédiger » $17 \%$, « reformuler » $13 \%$, « formuler » $11 \%$, « définir » $11 \%$, « réécrire » $8 \%$, « faire une synthèse » $6 \%$ et « faire une analyse » $2 \%$.

*53\% des activités de reformulation sont réalisées par le moyen de simplification.

*Dans les boîtes d'aide, $97 \%$ des reformulations sont effectuées par le biais de synonymie, 3\% par l'explication, la définition, la nominalisation, la dérivation et l'exemplification.

*Dans les boîtes de "préparation à l'écoute ", $85 \%$ d'actes de reformulation se fait par le biais de définitions, le reste se fait par le biais de synonymies et d'explications.

Pour conclure, on peut dire que, en particulier dans les pays non francophones, les manuels, qui sont connus pour « jouer un rôle primordial » dans l'enseignement des langues étrangères, ne sont pas que des contenus de simples exercices, instructions et autres activités grammaticales continues collectés dans un manuel "sans vie », mais, qu'ils visent à améliorer la capacité à utiliser la langue dans différents contextes "vivants », en tenant compte de la culture et du processus d'apprentissage cognitif de l'apprenant. Le livre Panorama 4, basé sur les principes de l'approche communicative fait souvent appel à l' " acte de reformulation ", considéré comme une créativité langagière à part entière. Il va sans dire que l'enseignement des langues étrangères n'est pas seulement dispensé en classe grâce à la communication entre les apprenants et l'enseignant, mais que les manuels utilisés dans le cours jouent également un rôle « important » dans l'enseignement des langues étrangères. L'apprenant, qui vise à acquérir 4 compétences linguistiques en reconstruisant ses connaissances, ses savoirs acquis dans différents contextes, améliorera également la qualité, ainsi que la quantité de ses compétences. Puisque les auteurs du manuel ont choisi principalement les moyens de «simplification», «synonymie» et «définition» lors de la mise en œuvre de l'«acte de reformulation», on peut dire que ce manuel s'appuie sur « un apprentissage qui part du connu pour aller vers l'inconnu », sur la mise en place de nouvelles structures basées sur les mots et les structures avec lesquels l'apprenant est déjà familiarisé, et nous montre qu'il comprend des activités qui demandent à l'apprenant de reconstruire, de reformuler ses acquis dans des contextes et styles différents. Les résultats de cette recherche révèlent une fois de plus que la langue n'est pas un ensemble de règles statiques et limitées, au contraire, c'est une structure, constamment reconstruite, vivante et active en matière de syntaxe, de sémantique, de lexicologie, etc. Que l'on peut résumer en « ce n'est pas parce qu'une personne connaît par cœur le dictionnaire d'une langue qu'il saura parler cette langue ».

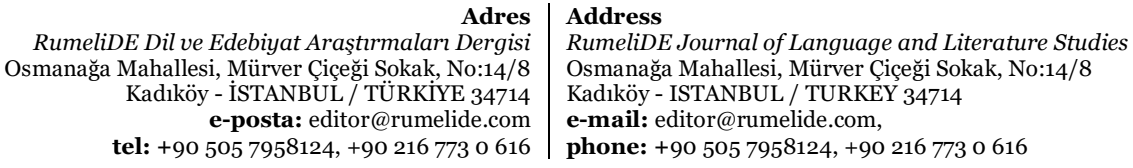




\section{Bibliographie}

Cuq, J. Et Gruca, I. (2005). Cours de didactique du français langue étrangère et seconde. France: PUG.

Girardet, J. (2009). Panorama de la langue française 4. Paris: CLE International.

Henry, J. (2016). Reformuler, réécrire, recréer... : des concepts connexes à la verbalisation en traduction, Jacqueline, Volume 29, numéro 1, TTR, 29 (1).

Jacobson, R. (1963) Essais de linguistique générale. 1. Les fondations du langage. Paris: Les Éditions de Minuit.

Karpiska-Szaj, K. et Paprocka-Pıtrowska,U. (2010). La reformulation en tant que stratégie d'apprentissage d'une langue : vers son exploitation dans des situations non-ordinaires" in, Roczniki Humanistyczne, Tom LXII, zeszyt 10 - 2014 p.55-81, https://www.ceeol.com/search/article-detail?id=257791 [Consulté le 8 Juin 2021]

Martinot, C. (1994). La reformulation dans des productions orales de définitions et explications. (Enfants de maternelle), Thèse de doctorat, dir. Blanche-Noëlle Grunig, Université Paris VIII.

Noyau, C. (2010). Développer les capacités de reformulation chez les maîtres de l'école bilingue en contexte subsaharien" in Congrès Mondial de Linguistique Française-CMLF Linguistique Française. Congrès Mondial de Linguistique Française - CMLF 2010, p.553-571 http://www.linguistiquefrancaise.org/ [Consulté le 8 Juin 2021]

\section{Les sites internet consultés :}

https://www.larousse.fr/dictionnaires/francais

Adres
RumeliDE Dil ve Edebiyat Araştırmaları Dergisi Osmanağa Mahallesi, Mürver Çiçeği Sokak, No:14/8 Kadıköy - ISTANBUL / TÜRKIYE 34714 e-posta: editor@rumelide.com tel: +90 $5057958124,+902167730616$
Address

RumeliDE Journal of Language and Literature Studies Osmanağa Mahallesi, Mürver Çiçeği Sokak, No:14/8

Kadıköy - ISTANBUL / TURKEY 34714

e-mail: editor@rumelide.com,

phone: +90 505 7958124, +90 2167730616 